\title{
Reconstruction of the W mass and width at and above WW threshold at FCC-ee
}

\author{
Marina Béguin ${ }^{* 1 \dagger}$, Paolo Azzurri ${ }^{2}$ and Elizabeth Locci ${ }^{3}$ \\ ${ }^{1}$ CERN, CEA-Saclay, Université Paris-Sud \\ ${ }^{2}$ INFN, Pisa \\ ${ }^{2}$ CEA-Saclay \\ E-mail: marina.beguin@cern.ch, paolo.azzurri@cern.ch, \\ elizabeth.locciecern.ch
}

In the Standard Model theory, the mass of the $\mathrm{W}$ boson is predicted with an uncertainty of $4 \mathrm{MeV} / \mathrm{c}^{2}$ whereas the current experimental accuracy is of $12 \mathrm{MeV} / \mathrm{c}^{2}$. The present uncertainty is currently limiting the sensitivity to possible effects of new physics through the global fit of the electroweak observables. With more than $2 \times 10^{8} \mathrm{~W}$ pairs produced at the $\mathrm{W}$ threshold energy and above, the FCC-ee collider will be a $\mathrm{W}$ boson factory allowing for $\mathrm{W}$ mass measurement with unparalleled precision. The $\mathrm{W}$ mass can be directly measured at and above the threshold from the kinematic reconstruction of the $\mathrm{W}$-pair decay products. In addition, $e^{+} e^{-}$collisions offer the possibility to derive the $\mathrm{W}$ mass from the WW cross-section measured at the pair-production threshold. The precise measurement of the $\mathrm{W}$ mass and width, with both methods, is presented in the context of a future experiment at FCC-ee. A statistical uncertainty on the W mass below $1 \mathrm{MeV} / \mathrm{c}^{2}$ is expected and the experimental and theoretical systematic uncertainties must be reduced to match such a level of precision.

European Physical Society Conference on High Energy Physics - EPS-HEP2019 -

10-17 July, 2019

Ghent, Belgium

\footnotetext{
*Speaker.

${ }^{\dagger}$ On Behalf of the WG2 working group
} 


\section{Introduction}

With more than $2 \times 10^{8} \mathrm{~W}$-pairs produced, FCC-ee will be a WW factory. All this statistics offers a lot of opportunities for the WW diboson physics, such as the accurate measurements of the $\mathrm{W}$ mass and width from direct reconstruction or from a threshold-scan but also the precise measurement of other observables linked to the $\mathrm{W}$ such as the partial widths, the strong coupling constant, the CKM matrix or the gauge self-couplings.

The precise relation between $M_{W}, M_{H}$ and $M_{t}$ is a crucial test of the internal consistency of the Standard Model (SM) and any failure might reveal new physics. However, there are currently no deviations between the mass measurements and their theoretical predictions within their uncertainties. The $\mathrm{W}$ mass which is now predicted with an uncertainty of about $4 \mathrm{MeV} / \mathrm{c}^{2}$ whereas the experimental accuracy is $12 \mathrm{MeV} / \mathrm{c}^{2}$, appears as fundamental parameter, which accuracy must be improved by a factor 10 for testing the consistency of SM.

\section{W mass measurement at the WW threshold}

The W-pair production threshold is around $160 \mathrm{GeV}$ and the precise measurement of the $\mathrm{W}$ mass at threshold is achievable because of the rapid raise of the cross-section in this region. As shown on Figure 1, the variation of the $\mathrm{W}$ mass will introduce a shift on the cross-section, $\sigma$, while the variation of the width will change the slope of its lineshape. These sensitivities allow to measure only the $\mathrm{W}$ mass at a single point and to estimate simultaneously the mass and width with two or more data-taking points.

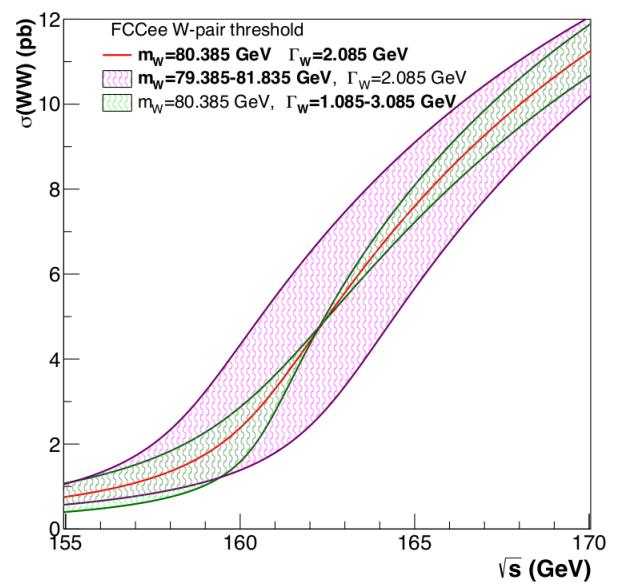

Figure 1: W-pair production cross-section variation with $\mathrm{W}$ mass and width as a function of the centre-of-mass energies [1].

The statistical uncertainty on the $\mathrm{W}$ mass is expressed as [1]:

$$
\Delta M_{W, \text { stat }}=\left(\frac{d \sigma}{d M_{W}}\right)^{-1} \frac{\sqrt{\sigma}}{\sqrt{\mathscr{L}}} \frac{1}{\sqrt{\varepsilon p}} \oplus\left(\frac{d \sigma}{d M_{W}}\right)^{-1} \frac{\Delta \sigma_{B}}{\varepsilon} \oplus\left(\frac{d \sigma}{d M_{W}}\right)^{-1} \sigma\left(\frac{\Delta \varepsilon}{\varepsilon} \oplus \frac{\Delta \mathscr{L}}{\mathscr{L}}\right) .
$$

$\varepsilon$ is the selection efficiency, $\mathscr{L}$ the luminosity, $p$ the purity defined as $\varepsilon \sigma /\left(\varepsilon \sigma+\sigma_{B}\right)$ and $\sigma_{B}$ is the background cross-section. The two last terms of Equation 2.1 correspond to the systematic uncertainties propagation of these observables to the $\mathrm{W}$ mass. To determine the $\mathrm{W}$ mass with the highest 
accuracy, the optimal data-taking point is chosen minimising the differential terms in Equation 2.1 as shown on Figure 2a. The optimal energy was found to be about $\sqrt{s}=161.4 \mathrm{GeV}$ which gives a statistical uncertainty on the $\mathrm{W}$ mass equals to $0.23 \mathrm{MeV} / \mathrm{c}^{2}\left(\mathscr{L}=12 \mathrm{ab}^{-1}\right)$. At LEP2, the statistical uncertainty on the $\mathrm{W}$ mass with the threshold-scan strategy was $210 \mathrm{MeV} / \mathrm{c}^{2}\left(\mathscr{L}=10 \mathrm{pb}^{-1}\right)$. Such precision requires the control of systematic uncertainties and is achievable only if the background cross-section is estimated at $10^{-3}$, the efficiency, luminosity and theoretical uncertainties with a $10^{-4}$ precision and the centre-of-mass energy is measured with a precision of $10^{-6}[1]$.

The same procedure can be used to determine simultaneously the mass and the width [1]. The optimal energies for the data-taking are found minimising the sensitivity of the cross-section to both the mass and the width as shown on Figure $2 \mathrm{a}$ and $2 \mathrm{~b}$. An optimal combination was evaluated to $E_{1}=157.1 \mathrm{GeV}, E_{2}=162.3 \mathrm{GeV}$ and $f=0.4$, where $f$ is the fraction of integrated luminosity attributed to $E_{1}$. With this scan optimisation, the uncertainties on the mass and width are $0.4 \mathrm{MeV} / \mathrm{c}^{2}$ and $1.2 \mathrm{MeV} / \mathrm{c}^{2}$ respectively.

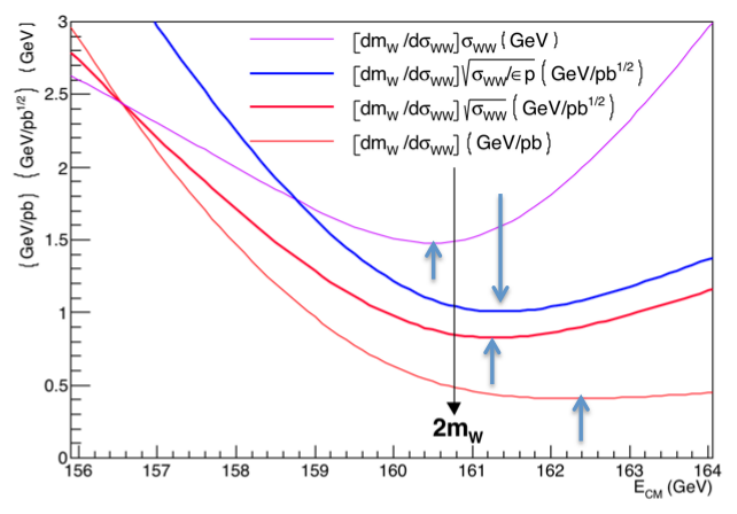

(a)

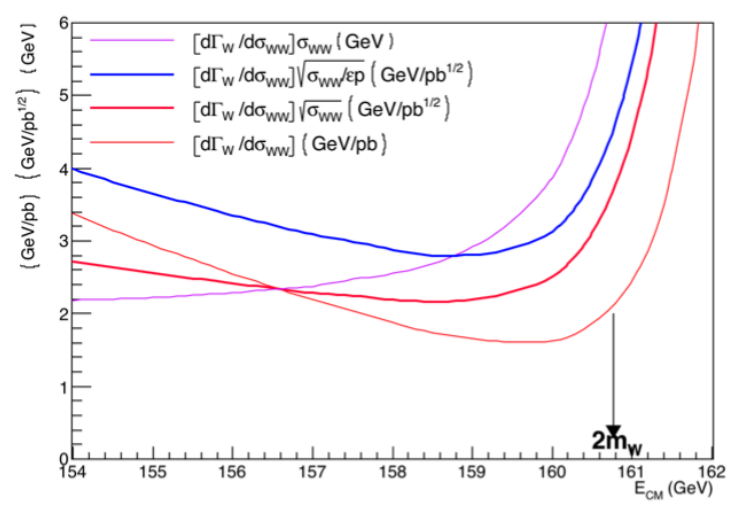

(b)

Figure 2: Sensitivity of the W-pair cross-section to the W mass (2a) and width (2b) as a function of $\sqrt{s}$ [1]. These distributions were evaluated with $\varepsilon=0.75, \sigma_{B}=0.3 \mathrm{pb}$ and $\mathscr{L}=12 \mathrm{ab}^{-1}$ and using YFSWW3 1.18 [2].

In order to calibrate the beam energy by resonant depolarisation, the collider must operate at energies corresponding to half-integer spin tune. Then the beam energy will be given by $E_{b}=$ $0.4406486(v+0.5) \mathrm{GeV}$ [3]. The optimal combination is therefore shifted to $E_{1}=157.3 \mathrm{GeV}$, $E_{2}=162.6 \mathrm{GeV}$ and $f=0.4$, changing the statistical uncertainties to $0.45 \mathrm{MeV} / \mathrm{c}^{2}$ and $1.3 \mathrm{MeV} / \mathrm{c}^{2}$ for the $\mathrm{W}$ mass and width respectively [1].

In the threshold-scan measurement, the energy spread is an important parameter to consider because it might shift $\sigma$ and introduce a systematic uncertainty on the $\mathrm{W}$ mass. The effect of the energy spread $\left(\sigma_{E}\right)$ is [3]:

$$
\delta \sigma_{W} \sim \frac{1}{2} \frac{d^{2} \sigma_{W}}{d E^{2}} \sigma_{E}^{2}
$$

At the FCC-ee, $\sigma_{E}$ will be measured/monitored with $e^{+} e^{-} \rightarrow \mu^{-} \mu^{+}$events with a relative precision of better than $0.2 \%$. It was shown that a relative precision of $1 \%$ has a very small effect 
on the cross-section, then it can be assumed that the energy spread has a negligible contribution on the $\mathrm{W}$ mass and width [3].

\section{Direct reconstruction of $M_{W}$ and $\Gamma_{W}$}

\subsection{Statistical uncertainties}

The other method to measure the $\mathrm{W}$ mass and width is the direct reconstruction, where the invariant mass (raw mass) is directly determined from the measured four fermion-momenta. Thanks to the huge statistics expected, this method can be used at all FCC-ee energies. The study was done at $162.6 \mathrm{GeV}, 240 \mathrm{GeV}$ and $365 \mathrm{GeV}$.

The hadronic and semi-leptonic decays of the $\mathrm{W}$ pair were simulated with the PYTHIA8 [4] event generator and propagated through the CLIC-like detector (CLD) [5] with HEPPY [6], a fast simulation software implemented for the FCC-ee detectors study. The decay products were forced into four jets using the DURHAM [7] algorithm in the hadronic channel and into two jets and a lepton in the semi-leptonic channel. Only the muon decay of this channel has been studied ${ }^{1}$.

The raw mass was improved using the kinematic fit technique $[1,11]$. The four-jets momenta were corrected imposing the energy-momentum conservation, corresponding to a four-constraint (4C) fit in the case of fully hadronic events and one-constraint (1C) fit in the case of semi-leptonic events. To further improve the invariant mass resolution, the mass equality of the two pair-produced bosons was required, for a five-constraint (5C) (two-constraint (2C)) fit for the hadronic (semileptonic) channel. At threshold, mass equality was not required because one of the two $\mathrm{W}$ masses is off-shell. In the hadronic decay channel, a jet four-momenta rescaling (4C rescaling) was used for a primary improvement of the invariant mass resolution, satisfying the total energy-momentum conservation while keeping jets angles and velocity fixed to their measured values. This method cannot be used in the semi-leptonic channel because the momentum of the neutrino is already computed from energy-momentum conservation. Figure 3 shows the smaller invariant (hadronic) and leptonic (semi-leptonic) mass distributions reconstructed with the different estimators at $\sqrt{s}=$ $240 \mathrm{GeV}$. The same kind of distributions were obtained at the other energies [11].

The statistical uncertainties on the $\mathrm{W}$ mass and width were estimated independently with a binned maximum likelihood fit on the reconstructed $\mathrm{W}$ mass distributions, using templates with different nominal $\mathrm{W}$ mass and width values. Except for the 5C and $1 \mathrm{C}$ fits, where only one mass is reconstructed, the expected uncertainty was obtained by combination of the two statistical uncertainties. Figure 4 shows the W mass statistical uncertainty for the full FCC-ee luminosity obtained in both decay channels with the different estimators.

In both channels, the most constrained kinematic fit gives the best invariant mass reconstruction and the smallest uncertainty on the $\mathrm{W}$ mass with uncertainties around $1 \mathrm{MeV} / \mathrm{c}^{2}$ or below in the hadronic decay and close to the $500 \mathrm{keV} / \mathrm{c}^{2}$ level in the semi-leptonic decay. The same study

\footnotetext{
${ }^{1}$ The track momentum for electrons that have not undergone bremsstrahlung is more precisely measured by the tracker. Assuming that the bremsstrahlung photon can be properly associated to the track, same performance may be expected in the electron and the muon channels. With this assumption, this study is done with muons only in order to simplify the issue.
} 

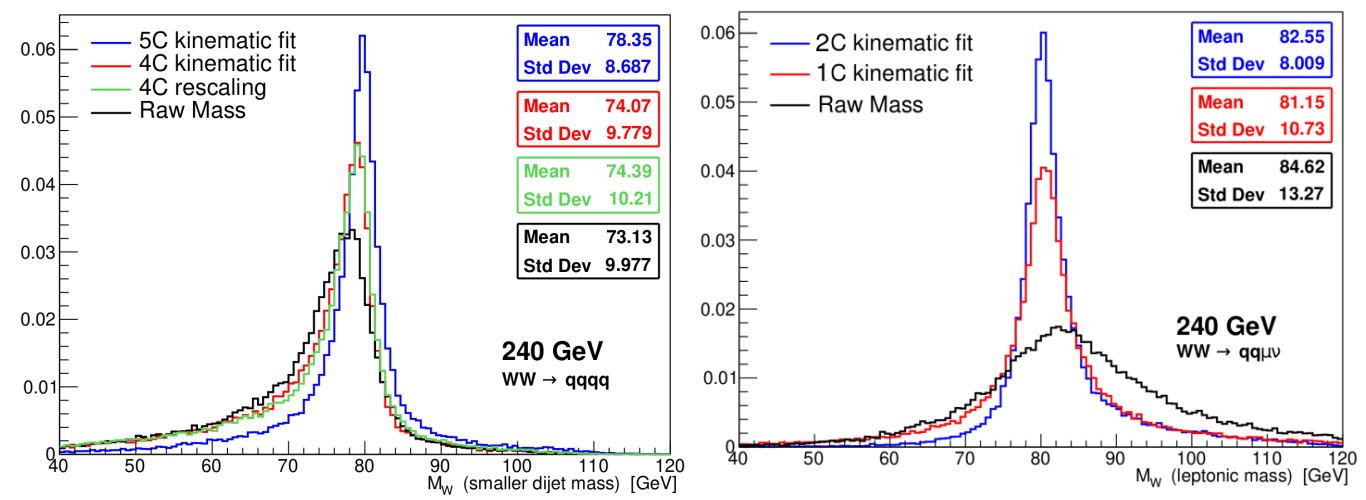

Figure 3: W invariant mass distributions in the hadronic (left) and semi-leptonic (right) channels reconstructed with different estimators at $\sqrt{s}=240 \mathrm{GeV}$.
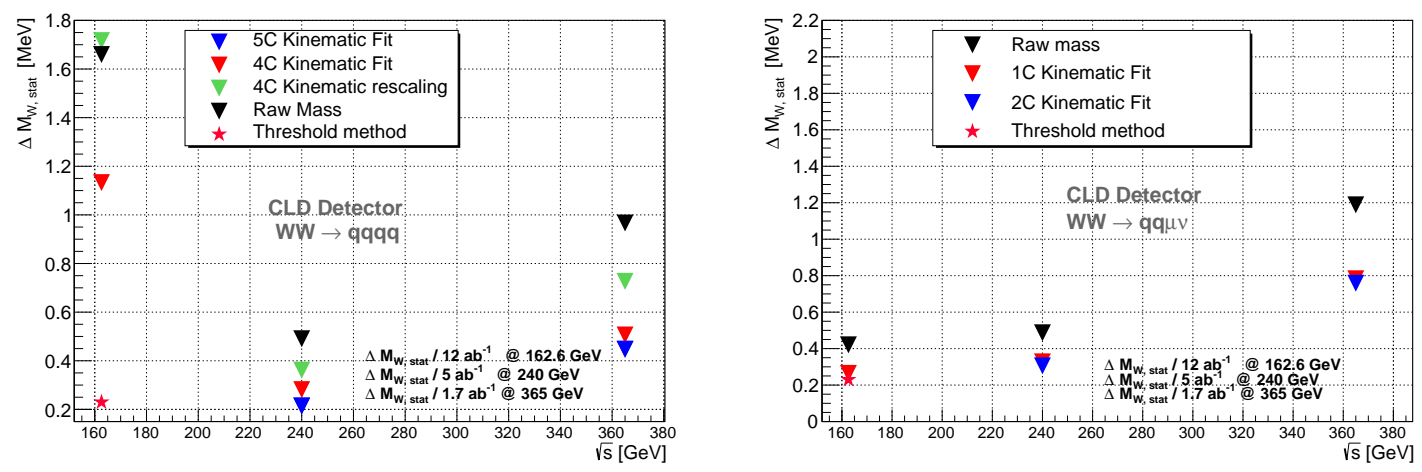

Figure 4: Estimated statistical uncertainties on the W mass in the hadronic (left) and semi-leptonic (right) channels reconstructed with different estimators at all centre-of-mass energies for the full FCC-ee luminosity.

\begin{tabular}{c|cc}
\hline \hline$\sqrt{s}[\mathrm{GeV}]$ & hadronic & semi-leptonic \\
\hline 162.6 & 1.1 & 0.35 \\
240 & 0.47 & 0.68 \\
365 & 1.0 & 1.56 \\
\hline \hline
\end{tabular}

Table 1: Estimated statistical uncertainty on the width $\left[\mathrm{MeV} / \mathrm{c}^{2}\right]$ evaluated with the most constrained fit in hadronic and semi-leptonic decay channels at different centre-of-mass energies.

was done for the width, the statistical uncertainties evaluated with the most constrained kinematic fit in both decay channels and all energies are shown in Table 1.

\subsection{Study of the systematic uncertainties}

Among the main sources of systematic uncertainties on the W mass at LEP2, the largest was due to the Final State Interactions (FSI): the Colour Reconnection (CR) and Bose-Einstein Correlations (BEC) [8]. Thanks to the huge statistics, the other sources of systematic uncertainties are expected to be largely reduced at FCC-ee, therefore only the FSI effects were studied. 


\begin{tabular}{ccccccc}
\hline \hline$\sqrt{s}[\mathrm{GeV}]$ & \multicolumn{2}{c}{162.6} & \multicolumn{2}{c}{240} & \multicolumn{2}{c}{365} \\
$\delta M_{F S I}\left[\mathrm{MeV} / \mathrm{c}^{2}\right]$ & standard & cone & standard & cone & standard & cone \\
\hline SKI & 14.6 & 7.5 & 23.9 & 11.5 & 32.2 & 17.5 \\
SKII & 7.9 & 3.8 & 12.1 & 6.0 & 14.7 & 8.3 \\
\hline BEC & 3.1 & 1.8 & 5.9 & 2.1 & 9.9 & 5.5 \\
\hline \hline
\end{tabular}

Table 2: Estimated systematic uncertainties introduced in the W mass by the CR (SKI and SKII models) and BEC in hadronic decay channel for different centre-of-mass energies.

The FSI were simulated with PYTHIA 8 and the CR was modelled using both the SKI and SKII models [9]. For the BEC study, one model, fitting all kinds of collisions, is implemented in PYTHIA [10]. To minimise the resulting shift of the $\mathrm{W}$ mass the ALEPH method was used: all particles outside a cone, directed around the jet axes and with an opening angle $\mathrm{R}=0.4 \mathrm{rad}$, were rejected. This opening angle value was chosen for a preliminary study of the FSI systematic uncertainty on the $\mathrm{W}$ mass. A more complete adjustment must be performed to determine the optimal value of the opening angle, which might differ at higher energies.

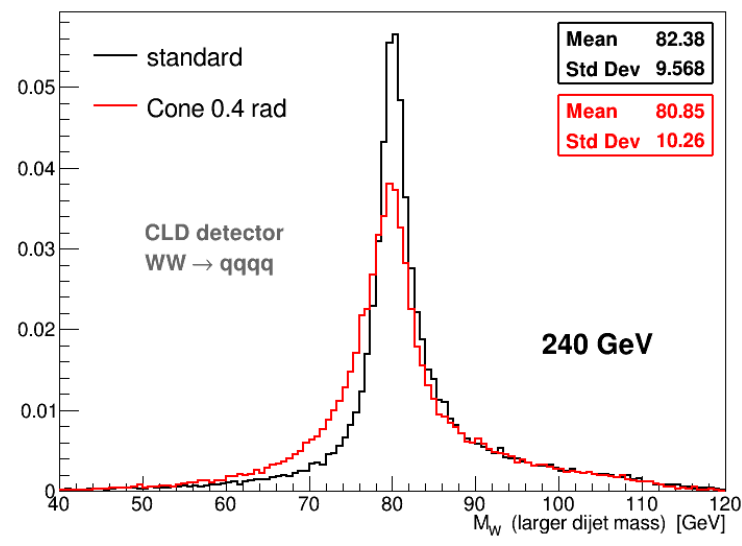

Figure 5: Invariant mass distribution in the hadronic channel (5C fit) with and without the cone at $240 \mathrm{GeV}$.

As shown on Figure 5, the loss of particle information because of the cone degrades the resolution. The degradations of the statistical uncertainties were estimated to be about a few percent at threshold, $10 \%$ at $240 \mathrm{GeV}$ and $15 \%$ at $365 \mathrm{GeV}$ [11]. Knowing that the cone should reduce the shift on the W mass by approximately $50 \%$ at all centre-of-mass energies (Table 2), the additional loss on the statistical uncertainties due to the cone is considered acceptable.

\section{Conclusion}

The amount of W-pairs at different centre-of-mass energies planned at FCC-ee presents a huge potential for the $\mathrm{W}$ physics measurements. This paper focuses on the $\mathrm{W}$ mass and width measurements with two different techniques. One of them relies on a threshold-scan strategy at the W-pair production threshold, where the mass and width can be accurately derived from the crosssection measurement. The other one is the direct reconstruction of the W-pair decay products at 
threshold and above, yielding to a statistical precision below the $\mathrm{MeV} / \mathrm{c}^{2}$ level. The study of the systematic uncertainty introduced by the FSI shows that it could be reduced with the cone treatment which was already used at LEP2.

Such precision on the $\mathrm{W}$ mass offers the possibility to measure the centre-of-mass energy with a good accuracy $\left(2 \mathrm{MeV} / \mathrm{c}^{2}\right.$ at $365 \mathrm{GeV}$ [3]) for energies above threshold, where the resonant depolarisation cannot be used. Ultimately, a simultaneous fit of WW, ZZ and Z $\gamma$ events could be performed to extract $m_{W} / m_{Z}$ with a potential large cancellation of the systematic uncertainties.

\section{Acknowledgments}

Many thanks to the EPS-HEP organisers for allowing me to give this talk as well as Alain Blondel, Patrizia Azzi, Markus Klute and Elizabeth Locci for the invitation. I would like to thank Elizabeth Locci for her help on both the presentation and the present document. Thanks to Paolo Azzurri for sharing his results on the $\mathrm{W}$ mass measurement at threshold (section 2. of this paper).

\section{References}

[1] P. Azzurri, M. Béguin and J. Gu, Di-boson physics, in preparation for submission (2019).

[2] S. Jadach and al., The Monte Carlo event generator YFSWW3 version 1.16 for W pair production and decay at LEP-2 / LC energies, Comput. Phys. Commun. $140432-474$ [hep-ph / 0103163 ].

[3] A. Blondel and al., Polarization and Centre-of-mass Energy Calibration at FCC-ee, JHEP [1909.12245].

[4] T. Sjöstrand, PYTHIA 8, Phys. Rept. [0809.0303].

[5] A. Abada and al., FCC Physics Opportunities, Eur. Phys. J. C79 (199-203) [10.1140/epjc/s10052-019-6904-3].

[6] C. Bernet, heppy, a generic event processing framework for high-energy physics https://github.com/cbernet/heppy.

[7] S. Catani and al., New clustering algorithm for multi-jet cross-sections in $e^{+} e^{-}$annihilation, Phys. Lett. B269 (432-438) [1 $0.1016 / 0370-2693$ (91) 90196-W].

[8] S. Schael and al., Electroweak Measurements in Electron-Positron Collisions at W-Boson-Pair Energies at LEP, Phys. Rept. 532 (119-244) [1302 . 3415].

[9] G. Gustafson and J. Häkkinen, Colour interference and confinement effects in W-pair production, Zeitschrift für Physik C Particles and Fields 64 (659-664)

[10] L. Lönnblad and T. Sjöstrand, Bose-Einstein effects and W mass determinations, Physics Letters B 351 (293 - 301).

[11] M. Béguin, Direct W mass measurement at and above threshold at FCC-ee, in preparation for submission. 